\title{
A mobile application for the stereoacuity test ${ }^{\star}$
}

\author{
Silvia Bonfanti, Angelo Gargantini, and Andrea Vitali \\ Department of Economics and Technology Management, Information Technology and \\ Production, University of Bergamo (BG), Dalmine, Italy \\ $\{$ silvia.bonfanti, angelo.gargantini, andrea.vitali1\}@unibg.it, \\ WWW project page: http://3d4amb.unibg.it
}

\begin{abstract}
The research paper concerns the development of a new mobile application emulating measurement of stereoacuity using Google Cardboard. Stereoacuity test is based on binocular vision that is the skill of human beings and most animals to recreate depth sense in visual scene. Google Cardboard is a very low cost device permitting to recreate depth sense of images showed on the screen of a smartphone. Proposed solution exploits Google Cardboard to recreate and manage depth sense through our mobile application that has been developed for Android devices. First, we describe the research context as well as aim of our research project. Then, we introduce the concept of stereopsis and technology used for emulating stereoacuity test. Finally, we portray preliminary tests made so far and achieved results are discussed.
\end{abstract}

\section{Introduction}

Having two eyes, as human beings and most animals, located at different lateral positions on the head allows binocular vision. It allows for two slightly different images to be created that provide a means of depth perception. Through high-level cognitive processing, the human brain uses binocular vision cues to determine depth in the visual scene. This particular brain skill is defined as stereopsis. Some pathologies, such as blindness on one eye and strabismus, cause a total or partial stereopsis absence. The examination of stereopsis ability can be evaluated by measuring stereoscopic acuity. Stereoscopic acuity, also named stereoacuity, is the smallest detectable depth difference that can be seen by someone with two eyes and normal brain functions.

Testing the total or partial loss of stereovision can lead to the detection of visual diseases like amblyopia. Amblyopia, otherwise known as 'lazy eye', is reduced visual acuity that results in poor or indistinct vision in one eye that is otherwise physically normal. This condition affects $2-3 \%$ of the population, which equates to conservatively around 10 million people under the age of 8 years worldwide [7]. Children who are not successfully treated when still young (generally before the age of 7) will become amblyopic adults. The projected lifetime risk of vision loss for an individual with amblyopia is estimated around at least $1-2 \%$ [5]. For these reasons, screening for amblyopia in early childhood is

\footnotetext{
* This work is partially supported by GenData 2020, a MIUR PRIN project.
} 
done in many countries to ensure that affected children are detected and treated within the critical period. The main goal is to help children to achieve a level of vision in their amblyopic eye that would be useful should they lose vision in their non-amblyopic eye later in life.

However, classical stereoacuity tests suffer from many problems: they are rather costly and can be performed only be specialized personnel. Moreover, they have a low level of sensitivity (vision problems may go undetected) and low specificity (vision problems are falsely reported).

For these reasons, we have been working on developing an efficient and affordable test, using for instance personal computer and $3 \mathrm{D}$ systems as in $[2,3,6,4]$. The system we proposed in [4] is still rather demanding in terms of equipment: it requires a desktop $\mathrm{PC}$ and a $3 \mathrm{D}$ vision system. In this paper we present a system which is composed by a simple Cardboard that realizes the $3 \mathrm{D}$ vision and whose cost is negligible, an Android smartphone, and a simple app that we describe in this work. The proposed solution promises to be simple to use, affordable, and precise.

The paper is organized as follows. In Sect. 2 we present some background regarding the use of $3 \mathrm{D}$ technologies for the virtual reality and we explain how the google Cardboard works. In Sect. 3 we introduce the stereoscopic acuity and some classical tests normally used to test and measure it. Sect. 4 introduces the mobile application we have developed in order to perform the stereoacuity test. Some preliminary results are presented in Sect. 5 and future works are discussed in Sect. 6.

\section{Virtual reality}

In the last years, many researchers have been involved in research works to design IT solutions to make more realistic the experiences with a $3 \mathrm{D}$ virtual environment. The aim is to permit users interact with virtual environments as done in real world. At this end, a set of devices have been developed to emulate the most important human senses which are tact and eyesight. There are devices that permit to track hands/fingers, such as Leap Motion device, Duo3D and Intel Gesture Camera (Fig. 1). These devices allow interacting with 3D objects using hands/fingers gestures and thin objects held in hand. These solutions can be used for medical applications such as design for lower limb prosthesis [1]. 3D systems have been developed to recreate depth sense on PC screen using LCD shutter glasses, such as NVIDIA@ 3D Vision ${ }^{\mathrm{TM}}$ system and Google Cardboard. 3D systems work in this way: they provide the two eyes with two different images of the same scene with a slightly offset viewing angles which correspond to the different viewpoints of our left and right eye. This vision produces an illusion of real depth of the scene and it is the basis of the $3 D$ virtual reality.

On computers and TVs, this effect is generally obtained by requiring the users to wear a special type of glasses, which are capable of separating the images display on the screen in two separate scenes for the left and right eye. The NVIDIA@ 3D Vision ${ }^{\mathrm{TM}}$ technology, which we used in another similar work 


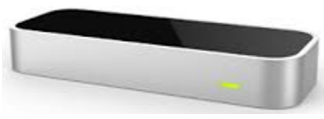

(a) Leap motion device

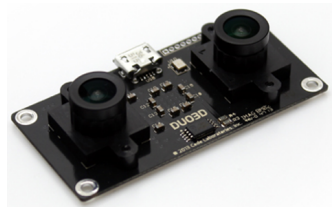

(b) Duo3D device

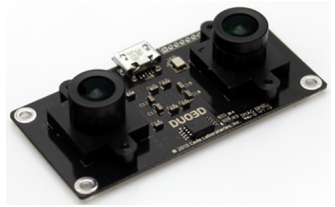

(c) Intel Gesture Camera

Fig. 1: Devices to track hands/fingers

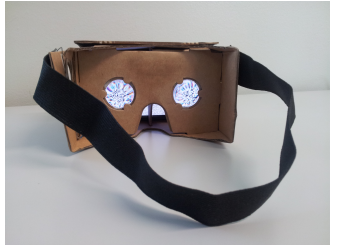

(a) Google Cardboard

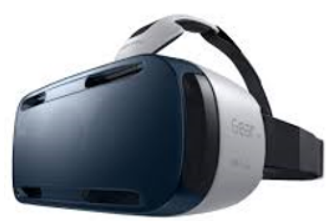

(b) Samsung Gear VR

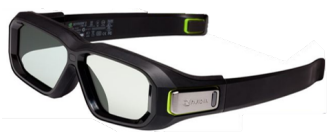

(c)

NVIDIA@ $3 \mathrm{D}$

Fig. 2: 3D devices

[4], requires a standard personal computer with a NVIDIA graphic card (also entry level NVIDIA graphic boards work), a monitor 3D Vision ready, which is capable of a refresh rate of $120 \mathrm{Mhz}$, and a NVIDA 3D glasses. The monitor alternates images for the two eyes and the glasses are able to synchronize the display of the images shown by the monitor in order to 3D glasses with their synchronizing emitter to be connect either to the USB port of the PC or directly to the graphic board. The Google Cardboard (Fig. 2a) is a simple box with two lenses that used in combination with a smartphone, constitutes a simple yet powerful virtual reality viewer. The smartphone must be inserted in the box and the user must watch inside in order to see the images displayed by the phone. It permits a stereo vision by sending two different images to the two eyes. It works with different smartphones and can be easily adapted to be used by children. The system proposed in this paper would work also with other types of VR viewers that use as display a smartphone (e.g., Samsung Gear VR).

In this research work, we pay our attention about 3D technology, which allows to simply emulate stereo vision. 3D vision systems are used to study different eye diseases, such as amblyopia and measurement of stereoacuity. About the use of virtual reality, IBit and our reserach project, i.e. 3D4Amb, are two research groups who are exploiting NVidia 3D technology in order to nurse amblyopia disease on young children. Moreover, 3D4Amb has started to develop a set of PCdesktop applications to emulate different visual tests that are usually performed by oculists and orthoptists, such as Lang test and stereoacuity test. In this paper, we present a new application to emulate stereo acuty test exploiting Google Cardboard to create depth sense through the use of an Android smartphone. 


\section{Stereoacuity measurement}

Stereoacuity is the smallest disparity that can be seen in binocular vision and it is measured in second of arc (arcsec). Stereoacuity tests can be divided into two groups: random dot stereotests and contour stereotests. Random dot stereotests are based on dots patterns arranged randomly with lateral disparity. These tests do not allow monocular vision. TNO, stereotest Lang I and II belong to this group. TNO is a test based on set of sheets with red and green random dots. Using red and green eyeglasses the patient is able to see $3 \mathrm{D}$ pictures if he is healthy. This test measures stereoacuity level from 2000 arcsec to 15 arcsec. Lang I and II are based on random dots and they do not need glasses because the dots are displaced to create disparity. These tests are mostly used in young children, but the lower measurable stereoacuity level is 550 arcsec in Lang I and 200 arcsec in Lang II. Contour stereotest are made up by two recognizable images, stagger, and shown to patients using polarized or anaglyph glasses. This category is not sensible as the former since the images are recognized also monocularly. Titmus stereotest belongs to contour stereotest and is based on vectographic technique. This test measures three levels of stereoacuity: high level around 3600 arcsec, medium level from 400 arcsec to 100 arcsec and low level until 40 arcsec. There is a test that is the union of random dot stereotests and contour stereotests: randot test. It is composed by two tables, the first is based on contour stereotest but background is replaced with dots. This technique prevents the monocular vision. Using this first table the measurable stereoacuity level is from 400 arcsec to 20 arcsec. The second table is based on random dot and it measures stereoacuity from 500 arcsec to 250 arcsec. All these tests can generate false negative because they have the following disadvantages:

- shown images are always the same, so the patients can memorize them and give right answers also if they do not see the right image;

- children can be helped by parents or doctors, so the test is not truthful.

In order to reduce the number of false negative we have devised the following policies:

- the shape is randomly chosen every time;

- the user that delivers the test has no clue about which shape is currently displayed.

Recently a new stereoacuity test was developed: 3DSAT [4]. This test is

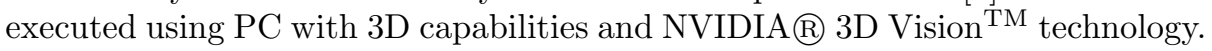
It is based on randot test and there is not monocular vision. During this test the images change always without a logical order and only the patient can see them. This increase the results truthfulness because none can help the patient.

\section{Stereoacuity Test App}

The application we present in this paper is called "StereoAcuity Test" and it is developed for Android devices. This test is based on randot stereoacuity test. 


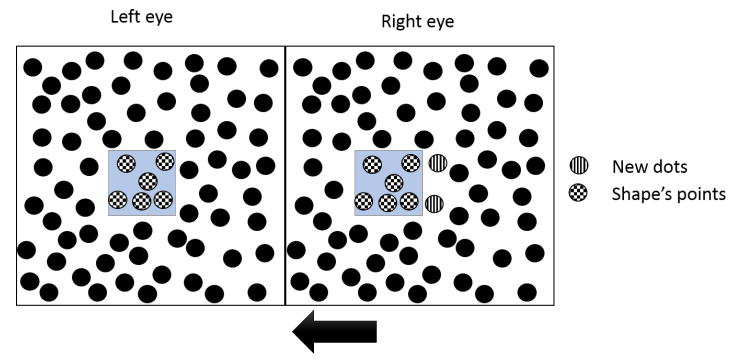

Fig. 3: Randot: principle of operation

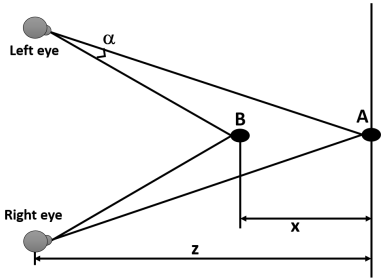

(a) Stereoacuity angle

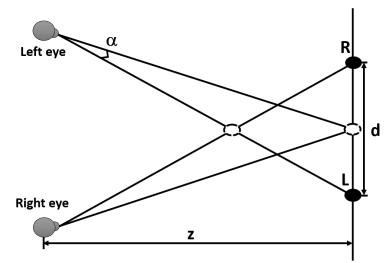

(b) Angle by virtual images

Fig. 4: Howard-Dolman Stereoacuity angle and angle by virtual images

The smartphone screen is split in two parts. Each eye sees one set of dots: one for the left eye and one for the right eye. The left eye sees a random dot image, while the right one sees the same image except for the dots within the shape that must be guessed. The points inside the shape are horizontally shifted (leftward) by a desired number of pixel. The blank space to the right of the shape is replaced by other dots. Principle of operation is shown in Fig. 3.

\subsection{Angle measurement}

Stereoscopic acuity, also named stereoacuity, is the smallest detectable depth difference that can be seen by someone with two eyes and normal brain functions. Its measure was introduced by Howard and Dolman who explained stereoacuity with a mathematical model as shown in Fig. 4a. The observer is shown a black peg (A in the figure) at a distance z. A second peg (B), in front of it, can be moved back and forth until it is just detectably nearer than the fixed one. Stereoacuity is this difference $(\mathrm{x})$ between the two positions, converted into an angle of binocular disparity $(\alpha)$.

The same effect can be replicated by using a 3D virtual system (like the Cardboard) by creating two virtual points ( $\mathrm{R}$ and $\mathrm{L}$ ), each to be seen by one eye, translated by a distance $d$, as shown in Fig. 4b. The disparity distance $d$ can be converted into the angle of disparity $\alpha$ in radiants by the following equation:

$$
\alpha=\arctan \frac{d / 2}{z}
$$




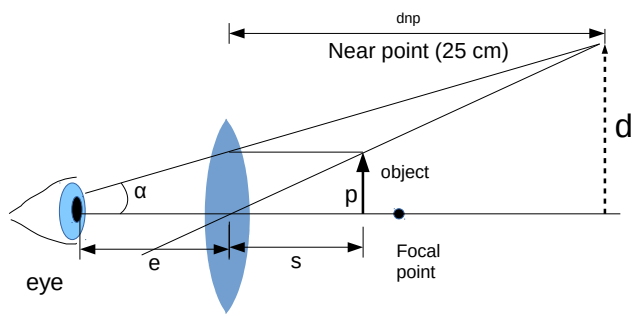

Fig. 5: Using a Cardboard

To convert $\alpha$ into the usual unit of seconds of arc, a multiplicative constant is used. The use of angles permits to evaluate the perceived dimensions of objects according to distance of the observer from the same objects. The stereacuity of humans is excellent when its value is around 60 seconds of arc.

In our case, we have to take into account the presence of the Cardboard lens. The Cardboard is provided with a positive lens with a short focal length (large dioptric power) to enlarge the image on your retina, much like the corrective lens for hyperopia does. In this way the images displayed on the phone appear in focus although the phone is very close to the eyes. This positive lens is usually referred to as a magnifying glass or a simple magnifier. It forms an enlarged image back at your near point distance for the eye, typically taken as 0.25 meters. Fig. 5 illustrates how the Cardboard lenses work.

A simple magnifier is characterized by its magnification power, usually denoted as a number with an mult, like $2 \mathrm{x}$. The magnification power is a measure of how much bigger the image appears with the magnifier than it does at the retina with the unaided eye. The calculation of the angle subtended by an object of height $p$ shown on the screen at distance $s$ from the lens, is the following:

$$
\alpha=\arctan \left(\frac{p}{s} d_{n p} \frac{1}{2} \frac{1}{e+d_{n p}}\right)
$$

Given a disparity in terms of pixels, for which one can compute the dimension of the shown object $p$ by considering the phone pixel density, the distance $e$ of the eye from the lenses, the distance $s$ of the phone from the lenses in the Cardboard, and the $d_{n p}$, then the formula above permits to obtain the angle $\alpha$.

Table 1 reports some configuration examples with selected smartphones and pixel disparities assuming a value for the eye distance of $5 \mathrm{~mm}$. As shown in the table, the angle can vary from around 1500 to min 120/150 seconds of arc for existing smartphones. The minimum angle is obtained by a disparity of 1 pixel. With higher pixel density screens, the test can reach smaller angles; for instance, with a new generation of screens capable of around 800 pixels per inch, the minimum angle will become around 78, which represents a typical excellent stereoacuity. 


\begin{tabular}{|c|c|c|c|c|c|c|c|}
\hline SmartPhone & $\begin{array}{l}\text { Screen } \\
\text { size (") }\end{array}$ & $\begin{array}{r}\text { pix } \\
\text { width }\end{array}$ & $\begin{array}{l}\text { els } \\
\text { height }\end{array}$ & ppi & $\begin{array}{l}1 \text { pixel } \\
\text { in } \mathrm{mm} \mathrm{d}\end{array}$ & $\begin{array}{l}\text { pixel } \\
\text { parity }\end{array}$ & $\begin{array}{l}\text { angle } \\
\text { econds) }\end{array}$ \\
\hline \multirow{4}{*}{ Galaxy S5 } & \multirow{4}{*}{5,1} & \multirow{4}{*}{1920} & \multirow{4}{*}{1080} & \multirow{4}{*}{431,9} & \multirow{4}{*}{0,059} & 1 & 156,5 \\
\hline & & & & & & 2 & 312,9 \\
\hline & & & & & & 5 & 782,3 \\
\hline & & & & & & 10 & 1564,6 \\
\hline \multirow{4}{*}{ LG G3 } & \multirow{4}{*}{5,5} & \multirow{4}{*}{2560} & \multirow{4}{*}{1440} & \multirow{4}{*}{534,0} & \multirow{4}{*}{0,048} & 1 & 126,6 \\
\hline & & & & & & 2 & 253,1 \\
\hline & & & & & & 5 & 632,8 \\
\hline & & & & & & 10 & 1265,5 \\
\hline \multirow{4}{*}{ Meizu MX4 Pro } & \multirow{4}{*}{5,5} & \multirow{4}{*}{2560} & \multirow{4}{*}{1536} & \multirow{4}{*}{542,8} & \multirow{4}{*}{0,047} & 1 & 124,5 \\
\hline & & & & & & 2 & 249,0 \\
\hline & & & & & & 5 & 622,5 \\
\hline & & & & & & 10 & 1245,1 \\
\hline Samsung (planned, 2015) & 5,1 & 3840 & 2160 & 863,9 & 0,029 & 1 & 78,2 \\
\hline
\end{tabular}

Table 1: Stereoacuity angle for smart-phones and disparity

\subsection{How application works}

The activity proposed by the software is divided into two parts: training and test phases. They are accessible from the main screen of application shown in Fig. 6.

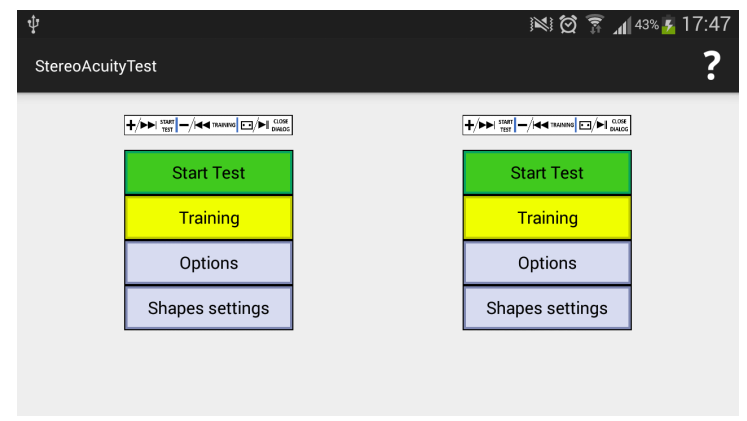

Fig. 6: Main screen

The training phase has been developed to make people understand how the test works and in which part of the screen they have to search the shape (Fig. 7a). The user has to force oneself to search the shape, but it is simplify since the figure has a different color compared to background. The patient can scroll shapes until he understands how use the application with Google Cardboard. After the training the user can start the test. The screen is split in two parts and it is overstuffed by points (Fig. 7b) inside which shapes are hidden. 


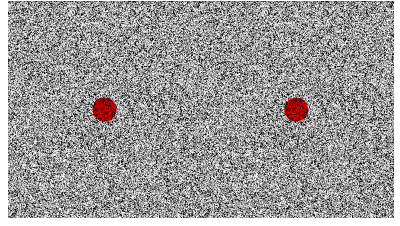

(a) Training screen

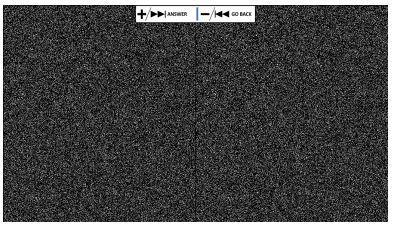

(b) Test screen

Fig. 7: Application screens

The patient watches inside the Cardboard until he finds the 3D shape. After that, using the iteration devices, he choose the image shown from a set of default forms (Fig. 8).

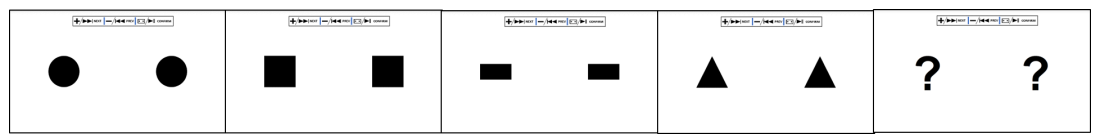

Fig. 8: Test answers

The user sees different shape with a decreasing level of stereoacuity. Stereoacuity level depends on the number of pixel of which shape is horizontally shifted. Higher is the number of pixel, higher is the stereoacuity level and the stereoacuity angle. He starts from the higher level and if he guesses the shape he runs down until he makes a mistake or guesses all levels. At this point of the test he certifies a stereoacuity level and the stereoacuity angle.

Furthermore it is possible to change the difficulty of test and shape settings from the main screen. "Shapes settings" button allows users to set the figures dimensions. Bigger are the shapes, easier is to discover them during the test. "Select test level" button contains options that entail level changing:

- Points dimension: is the dimension of the points generated on the screen. They are like 2 pixel per side as default.

- Points density: is the number of points generated on the screen to draw the shape and the background. Higher is this value greater is the number of points.

These parameters do not condition stereoacuity angle, they only increase the difficulty of guess the shapes.

\subsection{User iteration}

User iteration is not simple with this application since the smartphone is inside the Google Cardboard. There are two possible solutions: 


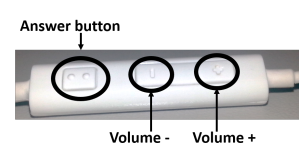

(a) Stereo headset

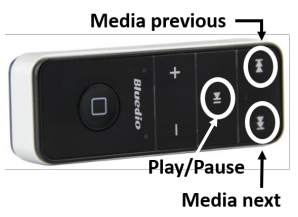

(b) Bluetooth stereo headset

Fig. 9: Instruments used by users

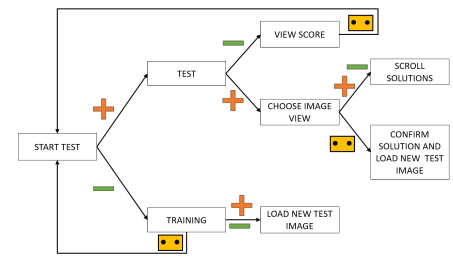

(a) Buttons used with stereo headset

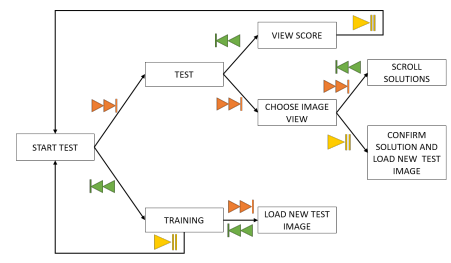

(b) Buttons used with bluetooth stereo headset

Fig. 10: User iteration

- stereo headset buttons (Fig. 9a)

- bluetooth stereo headset (Fig. 9b)

The stereo headset buttons used are volume + , - and answer button. The iteration diagram is shown in Fig. 10a. From the main screen it is possible to choose test phase (using + button) or training phase (using - button). Inside the training phase the patient can scroll all shapes using +/- volume buttons and return to main screen using answer button. In the test phase the patient uses + volume button to enter in the choose answer procedure, scrolls them using +/buttons and confirms using answer button. During the test user can interrupt it pushing - volume button. At the end of the test the application displays the score and it is possible to close it using answer button.

It is possible also use a bluetooth stereo headset (Fig. 10b). These buttons substitute stereo headset buttons with the following relation:

- volume $+=$ media next

- volume - = media previous

- answer button $=$ play $/$ pause

\section{Test results}

We performed some tests with the main aim of finding the best combination between points density and points dimension. Each test session is made up by seven levels and each level has different offset (number of leftward pixel). The 


\begin{tabular}{|c|c|c|c|c|c|c|}
\hline & \multicolumn{5}{|c|}{ Meantime to answer [sec] } & \\
Points dimension & User 1 & User 2 & User 3 & User 4 & User 5 & Meantime mean \\
\hline 1 pixel & 1,890 & 4,461 & 4,066 & 6,018 & 3,597 & 4,006 \\
\hline 2 pixel & 1,896 & 7,717 & 2,905 & 2,793 & 2,697 & 3,602 \\
\hline 3 pixel & 2,059 & 14,496 & 2,714 & 3,113 & 3,174 & 5,111 \\
\hline
\end{tabular}

Table 2: Meantime to answer with different points dimension and points density $=0.9$

user has to guess the shown shape. If the answer is right, then he carries on with the test until he reaches the first level, otherwise he finishes the test with a certain certified level. We have carried out tests to five users (without visual diseases) with different point dimensions and different point densities. For each level we measured the time to guess and the mean of all values is reported in Table 2 (only for point density $=0.9$ since the time to guess is lower). In the last column is computed the mean of all meantime for a certain point dimension. The lower value is obtained for point dimension equals to 2 pixel and this is set as default value in the application.

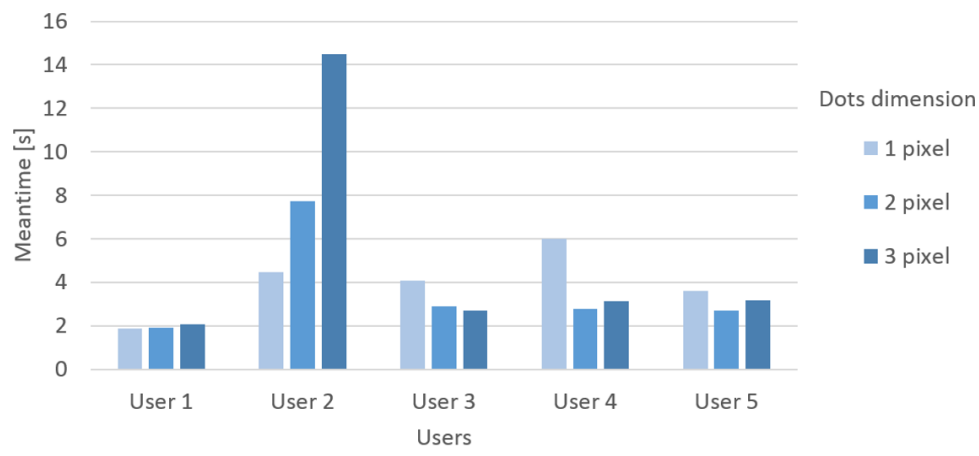

Fig. 11: Meantime to answer

\section{Future works}

At the moment the iteration between user and application is made by stereo headset buttons or bluetooth stereo headset (see Sect. 4.3). Feature work points to develop a twin application. The basic idea of this application is that the same application can be used by doctor and patient running on two different smartphones. Patient and doctor start the application on their smartphone and set the mode of operation. Doctor opens a listener port and waits until the patient smartphone sends a message. When the applications recognize each other the doctor can control the patient test without using stereo headset. At the end 


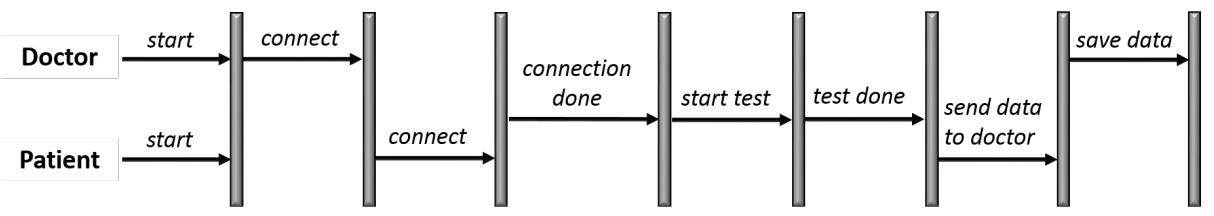

Fig. 12: Twin aplication

of the test, patient smartphone sends to the doctor test results and it saves all in a data base.

\section{Conclusions}

We have presented an Android application called "StereoAcuity Test" that emulates randot test using a smartphone and the Google Cardboard. Our test procedure ensures the accuracy of the results because only the patient sees the smartphone screen and recognize the images. Stereoacuity angles are a bit higher than some standard tests, but this is due to the pixel density of smartphones. We have demonstrated that if the pixel density increases, the stereoacuity angle decreases. Future steps will be the following:

- developing a twin application able to control the test through another smartphone

- performing additional tests to collect more data.

\section{References}

1. Giorgio Colombo, Giancarlo Facoetti, Caterina Rizzi, and Andrea Vitali. Socket virtual design based on low cost hand tracking and haptic devices. In Proceedings of the 12th ACM SIGGRAPH International Conference on Virtual-Reality Continuum and Its Applications in Industry, VRCAI '13, pages 63-70, New York, NY, USA, 2013. ACM.

2. Giancarlo Facoetti, Angelo Gargantini, and Andrea Vitali. An environment for domestic supervised amblyopia treatment. In VincentG. Duffy, editor, Digital Human Modeling. Applications in Health, Safety, Ergonomics and Risk Management, volume 8529 of Lecture Notes in Computer Science, pages 340-350. Springer International Publishing, 2014.

3. Angelo Gargantini, Mariella Bana, and Flavia Fabiani. Using 3D for rebalancing the visual system of amblyopic children. In Virtual Rehabilitation (ICVR), 2011 International Conference on, pages 1-7, june 2011.

4. Angelo Gargantini, Giancarlo Facoetti, and Andrea Vitali. A random dot stereoacuity test based on 3d technology. In Proceedings of the 8th International Conference on Pervasive Computing Technologies for Healthcare, PervasiveHealth '14, pages 358-361, ICST, Brussels, Belgium, Belgium, 2014. ICST (Institute for Computer Sciences, Social-Informatics and Telecommunications Engineering). 
5. Jugnoo S Rahi, Stuart Logan, Christine Timms, Isabelle Russell-Eggitt, and David Taylor. Risk, causes, and outcomes of visual impairment after loss of vision in the non-amblyopic eye: a population-based study. The Lancet, 360(9333):597-602, August 2002.

6. Andrea Vitali, Giancarlo Facoetti, and Angelo Gargantini. An environment for contrast-based treatment of amblyopia using 3D technology. In International Conference on Virtual Rehabilitation 2013 - August 26-29, 2013 in Philadelphia, PA, U.S.A., 2013.

7. Ann L. Webber and Joanne Wood. Amblyopia: prevalence, natural history, functional effects and treatment. Clinical and Experimental Optometry, 88(6):365-375, November 2005. 